\title{
Recommendations for prevention of weight gain and use of behavioural and pharmacologic interventions to manage overweight and obesity in adults in primary care
}

\author{
Canadian Task Force on Preventive Health Care*
}

See also CMAJ Open research articles, www.cmajopen.ca/content/2/4/E268 and www.cmajopen.ca/content/2/4/E306

Competing interests: See end of article.

This article has been peer reviewed.

*The complete list of authors appears at the end of the article. The complete list of current members of the Canadian Task Force on Preventive Health Care is available at canadiantask force.ca/about-us/members.

\section{Correspondence to:}

Canadian Task Force on

Preventive Health Care,

info@canadiantaskforce.ca;

Paula Brauer,

pbrauer@uoguelph.ca

CMAJ 2015. DOI:10.1503

/cmaj.140887
$\mathrm{T}$ he prevalence of obesity in adults has increased worldwide and has almost doubled in Canada, from $14 \%$ in $1978 / 79^{1}$ to $26 \%$ in $2009-2011,{ }^{2}$ with $2 \%$ of men and $5 \%$ of women having a body mass index (BMI) score greater than 40 (Appendix 1, available at www .cmaj.ca/lookup/suppl/doi:10.1503/cmaj.140887 /-/DC1). ${ }^{3}$ Over two-thirds of Canadian men (67\%) and more than half of Canadian women $(54 \%)$ are overweight or obese, based on measured weight. ${ }^{2-4}$ Excess weight is a well-recognized risk factor for several common chronic conditions, such as cardiovascular disease, cancer, type 2 diabetes, osteoarthritis and back pain. ${ }^{5}$

About two-thirds of adults who are overweight or obese were in the healthy weight range as adolescents, but gained weight in adulthood (about $0.5-1.0 \mathrm{~kg}$ every two years on average).,7 Slow weight gain may go unnoticed until health

\section{- Ker points}

Prevention of weight gain

- Body mass index is easy and inexpensive to measure, and can be used to monitor weight changes over time.

- Interventions for prevention of weight gain in adults of normal weight have a minimal effect and the effects are not sustained over time.

- Some individuals with normal weight may still benefit from interventions for weight-gain prevention, such as those with metabolic risk factors, high waist circumference, or a family history of type 2 diabetes or cardiovascular disease.

- For adults who are gaining weight and motivated to make lifestyle changes, practitioners should consider offering or referring to prevention interventions to prevent further weight gain.

Treatment of overweight and obesity

- Weight loss interventions (behavioural and/or pharmacologic) are effective in modestly reducing weight and waist circumference.

- Although most participants in weight-loss studies regain some weight after intervention, the average amount regained is lower among intervention participants than control participants.

- For adults who are at risk of type 2 diabetes, weight-loss interventions delay the onset of type 2 diabetes.

- Behavioural interventions are the preferred option, as the benefit-to-harm ratio appears more favourable than for pharmacologic interventions.

problems develop in middle age. However, this overall mean increase masks substantial variation in the population, and some people remain weight stable over long periods, whereas others experience substantial gains and losses. Currently, we cannot predict future health effects of weight gain over the life course.

The causes of obesity are complex. Although excess adiposity is ultimately the result of an imbalance in the amount of energy consumed and the amount of energy expended by an individual, there are many biological, behavioural, social and environmental factors that interact to affect this balance. The dramatic increase in obesity since the 1970s, however, suggests environmental causes are prominent and potentially amenable to interventions and prevention. ${ }^{89}$ Possible approaches include a wide range of legislative and policy options, in addition to health-promotion advice to maintain a healthy weight, increase physical activity and eat a healthy diet. ${ }^{10}$ Primary care providers have an important role in preventing and managing obesity through services offered to patients.

Overweight or obese status is commonly assessed using the BMI. The internationally recognized cut-off BMI values for adults are as follows: underweight $(<18.5)$, normal weight (18.5-24.9), overweight (25-29.9) and obese $(\geq 30)$. The obese category is further broken down by BMI into class I (30.0-34.9), class II (35.0-39.9) and class III ( $\geq 40) .{ }^{11}$ Distribution of body fat is also an independent indicator of health risk, and high waist circumference is associated with an increased risk for cardiovascular disease and type 2 diabetes. ${ }^{12}$

Lifestyle interventions (e.g., behavioural therapies, and dietary and physical activity programming and support) are the first line of treatment for most patients with overweight or obesity. Pharmacotherapy and surgery are options for more severe cases of obesity. A weight loss of $5 \%$ is considered clinically important, ${ }^{13}$ as many cardiovascular risk factors (e.g., elevated blood pres- 
sure, glucose and lipids) may improve with weight loss of this magnitude.

The objective of this guideline is to provide evidence-based recommendations for structured interventions aimed at preventing weight gain in adults of normal weight and to provide recommendations for behavioural and pharmacologic interventions for weight loss to manage overweight and obesity in adults, including those at risk of type 2 diabetes. Strategies for maintaining health, such as increased physical fitness, that did not emphasize or consider weight loss, were not reviewed. The guideline does not apply to people with a BMI score of 40 or greater, who may benefit from specialized bariatric programs. This document updates prior guidelines by the Canadian Task Force on Preventive Health Care, which were published in 1999 on treatment of obesity ${ }^{14}$ and in 2006 on prevention of obesity. ${ }^{15}$

\section{Methods}

The Canadian Task Force on Preventive Health Care is an independent panel of clinicians and methodologists that makes recommendations about clinical manoeuvres aimed at primary and secondary prevention (www.canadiantaskforce.ca). Work on each set of recommendations is led by a workgroup of two to six members of the task force. Each workgroup establishes the research questions and analytical framework for the guideline.

The development of these recommendations was led by a workgroup of task force members, in collaboration with scientific staff from the Public Health Agency of Canada (authors of guideline are listed at the end of the article). The workgroup established the research questions, and the analytical framework and clinically relevant outcomes for the guideline (Appendix 2, available at www.cmaj.ca/lookup/suppl/doi :10.1503/cmaj.140887/-/DC1), which were incorporated into the search protocol.

\section{Prevention of weight gain}

The task force initially developed an analytic model to assess effects of screening for obesity on health outcomes; however, no studies were found. The task force then made an informed decision to review the prevention literature separately from the treatment literature. The Evidence Review and Synthesis Centre at McMaster University independently conducted the systematic review on which these prevention recommendations are based, in accordance with the final, peer-reviewed protocol (Prospero \#CRD42012002753). ${ }^{16}$ The search included randomized controlled trials (RCTs) published between Jan. 1, 1980, and June 27,2013 , that were conducted in primary care set- tings or settings where primary care practitioners may refer patients. A clinical expert was consulted throughout the process.

After review of available outcomes, the task force chose to focus on the following clinically relevant and available outcomes: weight loss or weight maintenance (i.e., weight in kilograms, BMI, waist circumference, total percentage of body fat). Secondary outcomes of interest included total cholesterol, low-density-lipoprotein (LDL) cholesterol, fasting glucose, incidence of type 2 diabetes, systolic blood pressure and diastolic blood pressure (Appendix 2).

After the initial screening, there were insufficient RCTs that included only normal-weight participants. Yet, the evidence review centre identified numerous RCTs with a goal of preventing weight gain, but with study participants recruited from mixed-weight (i.e., normal-weight, overweight and obese) populations, which reflects clinical practice. The task force had the option to search for observational studies to address the first key question (i.e., do primary care-relevant prevention interventions [behaviourally based] in adults of normal weight lead to improved health outcomes or short-term or sustained weight-gain prevention, with or without improved physiologic measures?), or to use the evidence from the RCTs with mixed-weight populations. Given the potential for bias with lowerquality designs, the task force decided to use the indirect evidence from the RCTs to formulate the recommendations.

The evidence review focused only on intervention trials conducted in settings generalizable to Canadian primary care, feasible for conduct in primary care, or feasible for referral from primary care. Therefore, faith-based programs or studies conducted in specialist centres or educational settings were not reviewed.

\section{Management of overweight and obesity}

The research questions and analytical framework (Appendix 2) were based on the review of RCTs of behavioural and pharmacologic interventions for weight loss by the US Preventive Services Task Force. ${ }^{17}$ The Evidence Review and Synthesis Centre at McMaster University updated the 2011 search by the US Preventive Services Task Force to April 2013. ${ }^{17}$ All outcomes and analyses were determined a priori. There were sufficient studies to assess the primary outcomes of weight maintenance or loss, and secondary outcomes of improvements in blood pressure, glucose and lipid levels and incidence of type 2 diabetes. A clinical expert was consulted throughout the process.

The task force used the Grading of Recommendations Assessment, Development and Eval- 
uation (GRADE) system to determine the quality of evidence and strength of recommendations (Box 1). ${ }^{18}$ The recommendations were revised and approved by the entire task force and underwent external review by experts in the field and by stakeholders. More information about the task force's methods can be found elsewhere ${ }^{19}$ and at canadiantaskforce.ca/methods/methods-manual.

\section{Recommendations}

Box $2^{12}$ contains a summary of the recommendations. A summary of the GRADE decision table can be found in Appendix 3 (available at www .cmaj.ca/lookup/suppl/doi:10.1503/cmaj.140887 /-/DC1), with detailed tables provided in the accompanying evidence reviews..$^{16,22}$

\section{Measurement of BMI}

These recommendations apply to apparently healthy adults ( $\geq 18 \mathrm{yr}$ ) who present to primary care providers. The recommendations do not apply to people with eating disorders or who are pregnant. We recommend measuring height and weight and calculating BMI at appropriate primary care visits. (Strong recommendation; very low-quality evidence)

No studies of screening for weight issues in

\section{Box 1: Grading of recommendations}

- Recommendations are graded according to the GRADE system, ${ }^{18}$ which offers two strengths of recommendation: strong and weak. The strength of recommendations is based on the quality of supporting evidence, the degree of uncertainty about the balance between desirable and undesirable effects, the degree of uncertainty or variability in values and preferences, and the degree of uncertainty about whether the intervention represents a wise use of resources.

- Strong recommendations are those for which the task force is confident that the desirable effects of an intervention outweigh its undesirable effects (strong recommendation for an intervention) or that the undesirable effects of an intervention outweigh its desirable effects (strong recommendation against an intervention). A strong recommendation implies that most individuals will be best served by the recommended course of action. Weak recommendations are those for which the desirable effects probably outweigh the undesirable effects (weak recommendation for an intervention) or undesirable effects probably outweigh the desirable effects (weak recommendation against an intervention) but appreciable uncertainty exists.

- A weak recommendation implies that most people would want the recommended course of action but that many would not. For clinicians, this means they must recognize that different choices will be appropriate for each individual, and they must help each person arrive at a management decision consistent with his or her values and preferences. Policy-making will require substantial debate and involvement of various stakeholders. Weak recommendations result when the balance between desirable and undesirable effects is small, the quality of evidence is lower, or there is more variability in the values and preferences of patients.

- Evidence is graded as high, moderate, low or very low, based on how likely further research is to change our confidence in the estimate of effect.

Note: GRADE = Grading of Recommendations Assessment, Development and Evaluation primary care were identified. The measurement of height, weight and calculation of BMI is nevertheless recommended, because there is strong evidence that adults tend to overestimate height and underestimate weight, ${ }^{23}$ and there is increasing evidence that visual estimation by health providers is also relatively inaccurate. ${ }^{24}$ Thus, objective measurements are necessary to identify patients who are underweight, overweight and obese. It is also important to measure weight trajectories over time and intervene if people become overweight.

Of the clinically feasible measures, BMI is the body composition measure most strongly associated with mortality. ${ }^{25,26}$ Associations have been found to differ across populations, and lower cut-off points have been proposed in certain populations (e.g., South Asians), but the World Health Organization and other international groups continue to use the current cut-off points. ${ }^{27}$ More research and consultation is needed to establish appropriate BMI cut-off points for the major racial and ethnic groups in Canada, including Aboriginal populations. ${ }^{28}$

Other acceptable measures of obesity have been reviewed elsewhere and include waist circumference and waist-to-hip ratio. ${ }^{28-30}$ Waist circumference has been shown to independently classify individuals as higher risk for obesity-related illness, especially type 2 diabetes and cardiovascular diseases. ${ }^{31}$ Cut-off points for waist circumference vary across populations. ${ }^{27}$

Classifying and describing obesity by associated health problems and mortality risk (e.g., with the Edmonton Obesity Staging System) has been advocated, ${ }^{32}$ but trial results showing improved management and outcomes in primary care are required before such systems can be broadly recommended.

Despite its limitations, BMI was selected as the preferred measure because it is inexpensive and easy to apply (only height and weight are required to calculate BMI), no special training or equipment is required, and it can be used to monitor weight changes over time. Evidence of possible harms from prevention interventions was sought in the evidence review, but not specifically for calculating BMI.

The purpose of assessing BMI is to track changes in weight status over time, identify patients who may benefit from referral to obesity-management programs and to categorize patients with respect to overall cardiometabolic risk. For example, current diabetes screening recommendations suggest that BMI and waist circumference be used together to estimate the future risk of type 2 diabetes. ${ }^{12}$ Therefore, the task force recommends calcula- 
tion of BMI, placing a relatively high value on a low-cost, easily calculated measure with widely accepted cut-off points to base guidance for weight-gain prevention or management.

\section{Prevention of weight gain}

These recommendations apply to apparently healthy adults $(\geq 18 \mathrm{yr}$ ) of normal weight who present to primary care providers. The recommendations do not apply to people with eating disorders or who are pregnant, underweight (BMI $<18.5$ ), overweight (BMI 25-29.9) or obese $(\mathrm{BMI} \geq 30$ ).

\section{Interventions}

We recommend that practitioners not offer formal, structured interventions aimed at preventing weight gain in normal-weight adults. (Weak recommendation; very low-quality evidence)

A new systematic review of primary carerelevant RCTs comparing weight-gain preventions with no treatment was conducted to inform the recommendations. ${ }^{16}$ The systematic review of interventions for weight-gain prevention revealed only one study, ${ }^{33}$ published in 1988 , of an intervention to prevent weight gain exclusively in individuals of normal weight $(n=219)$ from the Minnesota Heart Health Program. Over the 12-month study, $82 \%$ of people in the intervention group (who received educational materials and a financial incentive to maintain weight) maintained or lost weight, compared with $56 \%$ in the control group, who received usual care $(p<0.0001)$. However, the mean difference in weight lost between the groups was minimal $(0.8 \mathrm{~kg}){ }^{33}$

The review identified evidence from 19 RCTs that focused on preventing weight gain in mixedweight populations (i.e., populations with normal-weight, overweight and obese adults) through lifestyle, diet and/or exercise, as compared with no intervention. ${ }^{16}$ Trials that enrolled only people with conditions predisposed to weight gain and associated adverse health effects (e.g., polycystic ovary or metabolic syndrome) or eating disorders were excluded from the systematic review. Trials of pharmacologic and surgical interventions were also excluded because these interventions were not considered relevant for prevention of weight gain among normal-weight individuals. To ensure the trials included some adults of normal weight, the number or percentage of normal-weight participants needed to be specified, or at least one study arm needed a baseline mean BMI or a baseline mean BMI minus one standard deviation that fell within the normal range (18.5-24.9). Power was likely sufficient in meta-analysis for assessment of the weight-change outcome ( $n=48460$ for all studies). Behavioural interventions (which generally included diet, exercise or other lifestyle components) lasted between 3 and 12 months to up to 12 years. ${ }^{16}$

\section{Box 2: Summary of recommendations for clinicians and policy-} makers

\section{Measurement of BMI}

This recommendation applies to adults ( $\geq 18 \mathrm{yr}$ ) presenting to primary care. These recommendations do not apply to people with eating disorders or who are pregnant.

- We recommend measuring height, weight and calculating $\mathrm{BMI}^{*}$ at appropriatet primary care visits. (Strong recommendation; very lowquality evidence)

\section{Prevention of weight gain}

This recommendation applies to apparently healthy adults ( $\geq 18 \mathrm{yr})$ who present to primary care. The recommendation does not apply to people with eating disorders, or who are underweight, pregnant, overweight or obese (BMI $\geq 25$ ).

- We recommend that practitioners not offer formal, structured interventions $\ddagger$ aimed at preventing weight gain in normal-weight adults.§ Adults who are overweight or obese may be candidates for weight-loss treatment. (Weak recommendation; very low-quality evidence)

\section{Management of overweight and obesity}

These recommendations apply to adults ( $\geq 18 \mathrm{yr}$ ) who are overweight or obese (BMI 25-39.9). Pregnant women and people with health conditions where weight loss is inappropriate are excluded. These guidelines do not apply to people with a BMI of 40 or greater, who may benefit from specialized bariatric programs.

- For adults who are obese (BMI 30-39.9) and are at high risk of diabetes, ๆ we recommend that practitioners offer or refer to structured behavioural interventions $\ddagger$ aimed at weight loss. (Strong recommendation; moderate-quality evidence)

- For adults who are overweight or obese, we recommend that practitioners offer or refer to structured behavioural interventions $\ddagger$ aimed at weight loss. (Weak recommendation; moderate-quality evidence)

- For adults who are overweight or obese, we recommend that practitioners not routinely offer pharmacologic interventions (orlistat or metformin) aimed at weight loss. ** (Weak recommendation; moderate-quality evidence)

Note: $\mathrm{BMI}=$ body mass index

*BMI categories are as follows: underweight (BMI < 18.5); normal weight (BMI 18.5-24.9); overweight (BMI 25.0-29.9); obese (BMI $\geq 30$ ).

†Appropriate visits include wellness visits, visits for medication renewal and other visits where the primary care practitioner deems it appropriate.

\#Formal structured interventions are behavioural modification programs that involve several sessions or interactions that take place over weeks to months. Interventions examined for prevention of weight gain included behaviourally based prevention interventions focused on diet, increasing exercise, making lifestyle changes or any combination of these. These could be offered in primary care settings or settings where primary care practitioners may refer patients, such as credible commercial or community programs. Recommended interventions for management of overweight and obesity include intensive behaviourally based interventions focused on diet, increasing exercise, making lifestyle changes or any combination of these. Lifestyle interventions generally included counselling, education or support, and/or environmental changes in addition to changes in exercise and/or diet.

§Practitioners should use their judgment in determining whether some individuals may benefit from being offered or referred to interventions for weight-gain prevention, such as individuals with metabolic risk factors, high waist circumference, or family history of type 2 diabetes or cardiovascular disease. For adults who express concerns about weight gain or who are motivated to make lifestyle changes, practitioners should also consider offering or referring to prevention interventions and must help each person arrive at a management decision consistent with his or her values and preferences.

THigh-risk status is defined by a 10 -year risk of diabetes of $33 \%$ or greater, ${ }^{12}$ which can be assessed using the CANRISK (Canadian Diabetes Risk) or FINDRISC (Finnish Type 2 Diabetes Risk Score) risk assessment tools. ${ }^{20,21}$

**The task force examined the use of metformin and orlistat for weight loss only and not for the treatment of other conditions, such as diabetes. 
Meta-analyses showed that intervention participants did not gain weight or gained less than controls. Overall, intervention participants lost an additional $0.7 \mathrm{~kg}$ more, lowered their BMI score by 0.2 more, reduced their waist circumference by an additional $1 \mathrm{~cm}$, and lost $1.3 \%$ more total body fat than control participants. ${ }^{16}$

Few long-term data exist to determine whether changes in weight are maintained after the interventions are completed. One study that did examine longer-term weight maintenance after promotion of physical activity in primary care found that initial weight changes were not sustained 15 months after the intervention. ${ }^{34}$ Overall reductions in total cholesterol, LDL and fasting glucose levels were $0.06,0.06$ and $0.04 \mathrm{mmol} / \mathrm{L}$, respectively; these are statistically significant but clinically small effects. No statistically significant reductions were found for systolic or diastolic blood pressure. Pooled results from two studies evaluating diabetes incidence in large mixed-weight populations $(n=$ $46537)^{35,36}$ suggest no effect of preventive interventions for obesity on diabetes incidence (risk ratio [RR] 0.95 , confidence interval [CI] 0.89 1.02). Three studies recruited people at higher risk of cardiovascular disease, whereas the rest included people at average, low or unknown risk of cardiovascular disease.

All 19 RCTs were considered to be very lowquality and were downgraded for risk of bias, indirectness and publication bias (details provided in the evidence review). ${ }^{16}$ The indirectness of the evidence relates to the fact that in 16 of the 19 studies, mean baseline BMI was in the overweight or obese range, and in 14 studies, participants in both treatment and control groups experienced modest weight loss. There was no evidence that patients with different baseline characteristics such as age, sex, cardiovascular risk or baseline BMI responded differently to the interventions. ${ }^{16}$

Whereas the goal of the reviewed studies was to avoid weight gain, and therefore the fact that the intervention participants did not increase their weight is relevant, the very low quality of the studies identified and their uncertain generalizability to normal-weight populations pose substantial limitations. No evidence indicated any harms associated with these preventive interventions. ${ }^{16}$ Insufficient evidence was available to determine patient values and preferences regarding interventions for weight-gain prevention. ${ }^{16}$

In making this recommendation against routinely offering or referring to interventions for weight-gain prevention, particularly in normalweight individuals, the task force carefully considered the evidence of limited effectiveness, the balance of potential benefits and harms, and potential resource implications, as outlined in the decision table for prevention of weight gain (Appendix 3).

This recommendation places a relatively high value on the importance of showing a clear net benefit before recommending programs for weight-gain prevention for the general population. Particular areas of concern were the lack of evidence of clinically meaningful prevention of short-term weight gain, the lack of evidence that weight was maintained over the long term, and the lack of evidence for effectiveness of preventive interventions in normal-weight populations specifically (as compared with mixed-weight populations, in whom benefits may differ). This recommendation places a relatively low value on the unproven possibility that programs for obesity prevention offered to the normal-weight population may reduce the long-term risk for obesity in that group.

However, the task force has offered a weak recommendation, because practitioners should use their judgment in determining whether some normal-weight adults may still benefit from being offered or referred to interventions for weight-gain prevention. Individuals who might be more likely to benefit from such referrals include those who are at higher risk of obesity or its consequences (e.g., type 2 diabetes or cardiovascular disease), or those who are highly motivated to avoid weight gain.

\section{Treatment of overweight and obesity}

Recommendations apply to adults ( $\geq 18 \mathrm{yr}$ ) who are overweight or obese (BMI 25-39.9). Pregnant women and people with health conditions where weight loss is inappropriate are excluded.

\section{Adults at high risk of diabetes}

For adults who are obese (BMI 30-39.9) and are at high risk of type 2 diabetes, we recommend that practitioners offer or refer to structured behavioural interventions aimed at weight loss. Structured interventions are intensive behavioural modification programs involving several sessions over weeks to months. Recommended interventions include behaviourally based interventions focused on diet, exercise or lifestyle changes, alone or in combination. Lifestyle changes include counselling, education or support, and/or environmental changes in addition to changes in exercise and/or diet. (Strong recommendation; moderate-quality evidence)

Differences in weight-related outcomes by risk groups only emerged in studies with participants at high risk of type 2 diabetes, defined based on pre-study glucose readings indicating impaired glucose tolerance ( $n=6$ studies) or val- 
idated risk assessment tools indicating high risk of diabetes ( $n=1$ study). Participants were less likely to receive a diagnosis of new-onset type 2 diabetes (11\% incidence) than control participants (20\%; RR $0.6,95 \%$ CI 0.4 to 0.7 ; absolute risk reduction $8.9 \%$ ). One of the largest studies showed that the risk of diabetes remained $34 \%$ (95\% CI 24 to 42) lower with intensive lifestyle changes than in the control group during a longterm $(10 \mathrm{yr})$ extension study. ${ }^{37}$

\section{Behavioural interventions}

For adults who are overweight or obese, we recommend that practitioners offer or refer to structured behavioural interventions aimed at weight loss. Structured interventions are as defined in the previous recommendation. (Weak recommendation; moderate-quality evidence)

Meta-analyses of trials with behavioural interventions (e.g., diet, exercise and/or lifestyle components) indicated that participants who received the intervention (baseline mean BMI of 31-32) lost on average $3.1 \mathrm{~kg}$ more than participants in the control group (95\% CI -3.9 to -2.4), lowered their BMI score by an additional 1.09 (95\% CI -1.4 to -0.8$)$, reduced their waist circumference by an additional 3.05 (95\% CI -3.9 to -2.2$) \mathrm{cm}$, and were more likely than controls to lose $5 \%$ or more (number needed to treat [NNT] 9; RR $1.8,95 \%$ CI 1.4 to 2.3 ) and $10 \%$ or more total body weight (NNT 12; RR 2.0, 95\% CI 1.3 to 3.2 ). ${ }^{22}$ Intervention participants also had significantly greater (although clinically small) reductions in levels of total cholesterol, LDL cholesterol and fasting glucose, and systolic and diastolic blood pressure than controls (Table 1). No trials reported on overall mortality or disease incidence, except for type 2 diabetes.

The trials included in the meta-analyses for this recommendation included participants with and without diabetes and other chronic conditions. Therefore, this recommendation applies to all populations, other than those at high risk for type 2 diabetes. People at high risk for type 2 diabetes are addressed in a separate recommendation.

In an analysis stratified by type of behavioural intervention, participation in diet alone ( $n=8$ studies), diet and exercise ( $n=10$ studies) and lifestyle ( $n=17$ studies) interventions led to greater reductions in weight of $4.7(95 \% \mathrm{CI}-6.2$ to -3.2$) \mathrm{kg}, 3.8$ (95\% CI -5.5 to -2.2$) \mathrm{kg}$ and 2.5 (95\% CI -3.5 to $-1.5) \mathrm{kg}$, respectively, than in controls. ${ }^{22}$ Exercise alone ( $n=4$ studies) was not associated with weight loss. Subgroup analysis by duration of intervention showed no difference based on duration.

Weight-gain prevention has been proposed as an alternative strategy for managing overweight and obesity. Results of the task force review informing the obesity prevention recommendations are relevant for those interested in strategies for weight-gain prevention. ${ }^{38}$ Despite the lack of evidence showing a clear net benefit, some overweight and obese adults may still benefit from being offered or referred to interventions for weight-gain prevention.

No studies were identified reporting significant harms of behavioural interventions. No significant difference in frequency of any adverse event, serious adverse event or withdrawal due to adverse events was reported between intervention groups and controls. ${ }^{22}$

\section{Pharmacologic interventions}

For adults who are overweight or obese, we recommend that practitioners not routinely offer pharmacologic interventions (orlistat or metformin) aimed at weight loss. (Weak recommendation; moderate-quality evidence)

All patients in the trials in the systematic review received dietary and/or exercise interventions, with the intervention group also receiving metformin or orlistat, and the controls receiving placebo. ${ }^{22}$

Meta-analysis showed that participants who received the intervention lost an additional 2.9 (95\% CI -3.5 to -2.3$) \mathrm{kg}$, lowered their BMI score by an additional 1.3 (95\% CI -1.8 to -0.7 ), reduced their waist circumference by an additional $2.3(95 \% \mathrm{CI}-3.0$ to -1.6$) \mathrm{cm}$, and were more likely to lose $5 \%$ or more (NNT 4; RR 1.8, 95\% CI 1.6 to 2.0 ) and $10 \%$ or more (NNT 8; RR $1.9,95 \%$ CI 1.7 to 2.2) of their total baseline body weight than controls (Table 1). The mean BMI score at baseline was 34-36 for patients in the pharmacologic trials. Both metformin $(n=2$ studies) and orlistat ( $n=15$ studies) were effective. ${ }^{22}$ Participants in the intervention group also had significantly greater reductions in levels of cholesterol, LDL cholesterol and fasting glucose, and systolic and diastolic blood pressure than the control group (Table 1). ${ }^{22}$

Three pharmacologic plus behavioural studies examined incidence of type 2 diabetes. Participants in the intervention group were less likely to receive a diagnosis of new-onset type 2 diabetes (RR 0.7, 95\% CI 0.6 to 0.9 ; absolute risk reduction $3.6 \%$ ) than those in the control group. ${ }^{22}$

Several concerns have led the task force to recommend against the routine use of pharmacologic treatments for management of overweight and obesity. Participants receiving the intervention in pharmacologic trials were more likely to experience adverse events (number needed to harm $[\mathrm{NNH}] 10)$, gastrointestinal events $(\mathrm{NNH}$ 5) and withdraw because of gastrointestinal and other harms (NNH 32), compared with those in the control group..$^{22}$ 
Most studies included a run-in period during which participants were required to reduce calories and encouraged to increase physical activity. In some cases, patients were stratified for inclusion based on the amount of weight lost during the runin period. Most studies provided no data on the proportion of participants excluded from trials after the run-in periods, and therefore the generalizability to the broader population is questionable.

Finally, all control groups received some behavioural intervention. Because of these concerns and the identified harms, the task force recommends against pharmacologic interventions for the management of overweight and obesity. Yet, pharmacologic therapy may be warranted in some situations. Physicians will need to consider the potential for benefits and harms in advising those patients who may benefit from the addition of pharmacologic therapy to behavioural change (e.g., individuals at risk for diabetes, or those who are highly motivated to lose weight and are less concerned about potential harms). As with behavioural interventions, personal values, preferences, experience and supports will be the main determinants of which interventions will be best suited for individual patients.

\section{Weight maintenance}

Two studies on behavioural interventions and four on pharmacologic plus behavioural interventions reported on weight maintenance. ${ }^{22}$

Partial weight regain was common, but par-

\begin{tabular}{|c|c|c|}
\hline \multirow[b]{2}{*}{ Outcome } & \multicolumn{2}{|c|}{ Intervention; mean difference $(95 \% \mathrm{Cl})$} \\
\hline & Behavioural & Pharmacologic \\
\hline \multicolumn{3}{|l|}{ Primary } \\
\hline Weight, kg & $-3.13(-3.88$ to -2.38$)$ & $-2.89 \quad(-3.49$ to -2.29$)$ \\
\hline BMI & $-1.09 \quad(-1.43$ to -0.75$)$ & $-1.27 \quad(-1.82$ to -0.72$)$ \\
\hline $\begin{array}{l}\text { Waist circumference, } \\
\mathrm{cm}\end{array}$ & $-3.05 \quad(-3.86$ to -2.24$)$ & $-2.29 \quad(-3.04$ to -1.55$)$ \\
\hline \multicolumn{3}{|l|}{ Secondary } \\
\hline $\begin{array}{l}\text { Total cholesterol, } \\
\mathrm{mmol} / \mathrm{L}\end{array}$ & $-0.10 \quad(-0.18$ to -0.03$)$ & $-0.33(-0.42$ to -0.24$)$ \\
\hline $\begin{array}{l}\text { LDL cholesterol, } \\
\mathrm{mmol} / \mathrm{L}\end{array}$ & $-0.14 \quad(-0.29$ to -0.00$)$ & $-0.28 \quad(-0.38$ to -0.19$)$ \\
\hline $\begin{array}{l}\text { Fasting glucose, } \\
\mathrm{mmol} / \mathrm{L}\end{array}$ & $-0.14 \quad(-0.23$ to -0.05$)$ & $-0.43 \quad(-0.66$ to -0.20$)$ \\
\hline $\begin{array}{l}\text { Systolic blood pressure, } \\
\mathrm{mm} \mathrm{Hg}\end{array}$ & $-1.76 \quad(-2.61$ to -0.91$)$ & $-1.70 \quad(-2.28$ to -1.13$)$ \\
\hline $\begin{array}{l}\text { Diastolic blood } \\
\text { pressure, } \mathrm{mm} \mathrm{Hg}\end{array}$ & $-1.60 \quad(-2.27$ to -0.93$)$ & $-1.24 \quad(-1.88$ to -0.61$)$ \\
\hline
\end{tabular}

ticipants in the two behavioural intervention studies regained less weight (mean difference $-1.6,95 \% \mathrm{CI}-3.1$ to $-0.02, \mathrm{~kg}$ ) than controls. Mean changes in the four maintenance interventions using orlistat found no effect on weight maintenance in kilograms of orlistat compared with control (mean difference $-1.4,95 \%$ CI -2.9 to $0.08, \mathrm{~kg}$ ). Maintenance interventions using orlistat showed improved maintenance of weight loss of $5 \%$ or more, but not of $10 \%$ or more; this outcome was not examined in the behavioural trials. Results are consistent with other estimates in the literature. ${ }^{39}$ Currently, it is expected that patients require ongoing encouragement to maintain healthy lifestyle behaviours, while accepting some weight regain.

\section{Balance of benefits and harms}

The task force places a higher value on the evidence for short-term weight loss without important harms and on improvements in certain secondary health outcomes with behavioural interventions. Given the large number of studies with consistent results, new studies of behavioural interventions are not expected to achieve substantially different results. The weak recommendation is due to the limited evidence on the long-term effectiveness of interventions on health indicators. For example, no data on clinically relevant outcomes, such as mortality, were identified.

The task force recommends against pharmacologic intervention to manage overweight and obesity, although some patients may prefer medications and be good candidates for pharmacologic treatment. Of note, all pharmacologic interventions in our review also included a behavioural component and therefore pharmacologic monotherapy (without the behavioural intervention) may not be as effective.

If treatment is implemented, patients should be aware that the magnitude of the average expected weight change is modest (about $3 \mathrm{~kg}$ ), although this change may be clinically meaningful, especially in those at higher risk of obesityrelated complications. For those at risk of type 2 diabetes, the potentially beneficial delay in the onset of diabetes was sufficient for the task force to offer a strong recommendation.

\section{Considerations for implementation}

The task force has developed a series of tools to help practitioners interpret these recommendations for their patients, which can be found at canadiantaskforce.ca. The task force used a rigorous and collaborative usability testing process to develop knowledge translation tools targeting clinicians to accompany this guideline. All tools 
are informed by feedback from clinicians. They have not been formally tested in practice.

\section{Patient values and preferences}

For adults of normal weight who express concerns about weight gain or who are motivated to make lifestyle changes, practitioners should discuss the evidence showing minimal short-term benefit from interventions for weight-gain prevention with their patients and should help each patient make a decision that is consistent with his or her values and preferences. Lifestyle changes focusing solely on increased physical fitness levels, or improved quality of life were not included in the examined trials, but there is increasing evidence that physical fitness modifies the relation between body weight and mortality. ${ }^{38}$

The evidence review on treatment of overweight and obesity found limited information on patients' values and preferences about treatment. ${ }^{22}$ Brown and Gould ${ }^{40}$ undertook a systematic review of qualitative studies reporting on perceptions, experiences, contexts and influences for adults facing, or reflecting on, weight management. The authors identified a range of factors that would influence the decision to undertake weight loss, including cultural identity, responses to obesity stigma, previous weight-loss experiences, personal motivators and barriers, social support and practical resources. ${ }^{40}$ Another review ${ }^{41}$ described similar motivators for weight-management programs (e.g., health concerns, mental preparedness, self-perception and body image, and past positive experiences). Sociocultural factors could be facilitators or barriers, depending on the individual and culture, and weight-management attempts were influenced by expectations of weight management. Most participants in both reviews were women, and thus the results may not be generalizable to men.

\section{Assessment of BMI and health risk}

Practitioners should use clinical judgment to decide the frequency with which patients should have their weight and health status assessed.

We recommend that a noninvasive, validated risk-assessment tool (e.g., CANRISK [Canadian Diabetes Risk] ${ }^{20}$ or FINRISC [Finnish Type 2 Diabetes Risk Score ${ }^{21}$ ) be used to calculate risk of type 2 diabetes in overweight and obese patients, as per recommendations for screening. ${ }^{12}$ (The tools are available at www.publichealth.gc .ca/CANRISK and canadiantaskforce.ca/ctfphc -guidelines/2012-type-2-diabetes/clinician-findrisc, respectively.) The current guidance on diabetes screening suggests that risk assessment be done at least every three to five years in people at high risk of diabetes developing within 10 years. ${ }^{12}$
Interventions for overweight and obesity

Practitioners should be aware of barriers to participation in weight-loss interventions, such as unrealistic expectations, hunger, knowledge and/ or skills, sociocultural factors, psychological problems, past stigmatizing experiences and environmental factors. ${ }^{22,40,41}$ Patients who have tried behavioural interventions without success may benefit from a greater focus on positive lifestyle changes, such as promotion of physical activity and weight-gain prevention. ${ }^{16,22}$

The most effective interventions were highly heterogeneous with respect to provider discipline, length and format. Therefore, a specific program cannot be recommended; however, the efficacious behavioural interventions tended to be of greater than 12 months duration, included diet and/or exercise and/or lifestyle components, and included group and individual sessions. ${ }^{11,22}$ These interventions are likely appropriate for patients who are ready and able to make substantive lifestyle changes. A separate meta-analysis of 24 pragmatic lifestyle interventions for prevention of diabetes found a mean weight loss of $2.1 \mathrm{~kg},{ }^{42}$ about twothirds of that achieved in the studies reviewed for this guideline.

\section{Other considerations}

There is evidence showing an association between obesity and socioeconomic status, and related factors such as education and income. ${ }^{43}$ One study in particular examined the variation in the relation between BMI and income across Canadian provinces and suggested it may be affected by regional availability, food prices and rates of income taxation. ${ }^{44}$ The systematic review for treatment of overweight and obesity indicated that the prevalence of obesity in Canada is higher in certain segments of the population. ${ }^{30}$ Prevalence is higher in Aboriginal people than in the remainder of the population, higher in white Canadians than in visible minorities, and higher in nonimmigrants than in recent immigrants $(<10 \mathrm{yr}$ in Canada). Therefore, although primary care practitioners have an important role to play in the prevention and management of overweight and obesity, a multisectoral solution is required to address the obesity epidemic.

Most studies in the systematic review for treatment of overweight and obesity were conducted in Europe $(n=31)$ or the United States $(n=26){ }^{22}$ The task force considers the results applicable to the Canadian population. The settings for interventions for overweight and obesity varied, and included primary care, community, home and multiple settings. The local context (i.e., availability in urban or rural areas, northern communities) should 
be considered when discussing behavioural or pharmacologic interventions with patients.

\section{Suggested performance indicators}

Given the limitations of the evidence, no performance indicators were developed for prevention of weight gain in adults of normal weight.

Performance measures for management of overweight and obesity include the proportion of adults with overweight or obesity (in particular those at risk of diabetes), in whom the weightloss interventions are offered or discussed, who participate in structured programs and who achieve weight loss. In the reviewed studies, $20 \%$ achieved a weight loss of 5\% and $11 \%$ achieved a weight loss of $10 \% .{ }^{22}$

\section{Economic implications}

Given the paucity of direct evidence for prevention, the task force did not evaluate the economic implications of interventions for prevention of weight gain in detail. The task force is also concerned about the intensity of the effective interventions. A qualitative assessment of the effective interventions for prevention of weight gain found that they tended to be of long duration ( $\geq 12 \mathrm{mo}$ ), involved personal contact with providers in individual sessions and in some cases group sessions, often included an exercise component, and generally involved multiple sessions, which implies a substantial commitment on behalf of both providers and patients.

We also did not consider the economic implications of interventions for weight loss in overweight and obesity, but we searched the literature on resource implications. Our review of the available literature found that adults with obesity have higher rates of health service use, ${ }^{22}$ and available data are inconsistent about whether behaviourally based obesity interventions are cost-effective. No Canadian modelling studies reflecting costs and effects over the long term were identified.

\section{Other guidelines}

The task force's last guidance on obesity prevention was in $2006 .{ }^{15}$ At that time, the task force recommended individual and small-group counselling for a reduced calorie or low-fat diet to prevent obesity, and/or an intensive individual or structured group program of endurance exercise to prevent obesity. The previous recommendations were based on evidence from 12 studies,,$^{15}$ whereas the current recommendations are based on a new review of 26 studies, only two of which were included in the 2006 review. Despite the fact that different studies were included in the new review, the current recommendations are based on similar evidence: the studies included participants from mixed-weight (i.e., normal-weight, overweight and obese) groups. In the case of overweight and obese individuals, the intervention may be considered treatment, not prevention. The new task force methods $^{45}$ (i.e., GRADE methodology ${ }^{18}$ ) highlight the indirectness and limitations of such evidence. When developing the new guidelines, the task force placed a high value on the lack of good-quality evidence showing the effectiveness of preventive interventions in the normal-weight population (Appendix 3) and therefore reached different conclusions regarding recommendations to prevent weight gain.

The recommendations of other groups on prevention of weight gain are shown in Table 2. The US Preventive Services Task Force has no guideline on the prevention of obesity and bases its screening for obesity recommendations on indirect evidence of the effectiveness of treatment interventions. ${ }^{46}$ The National Institute for Health and Clinical Excellence in the United Kingdom ${ }^{47}$ recommends healthy eating and increasing physical activity to improve an individual's general health, whereas the Canadian task force did not examine healthy eating and physical activity as general health-promotion interventions, only as interventions aimed at reducing or maintaining weight.

The task force previously recommended in favour of interventions for adults with obesityrelated diseases (e.g., diabetes, hypertension). ${ }^{14}$ Differences from previous task force recommendations are due to differences in methodology and grading of evidence, and updates in knowledge regarding management of overweight and obesity. The current task force guidance on management of overweight and obesity is consistent with international guidelines and strategies (Table 2).

\section{Gaps in knowledge}

Few studies exist that are designed to help patients of normal weight with or without specific health risks to maintain their weight. Such studies in both solo-practitioner and team-based care delivery models are needed. As such, this guideline was limited in its ability to comment on prevention of weight gain in normal-weight populations, because the data are largely based on trials from mixed-weight populations. As overweight and obesity rates continue to rise in Canada, it becomes increasingly important for practitioners to understand how adults of normal weight can best maintain their health over time, and whether structured interventions beyond standard advice for healthy living should be 
implemented with normal-weight populations. It is also important to better understand additional benefits in improved functioning and quality of life that could result from interventions. Research is needed on predictors of future health risk associated with weight gain. More research is also needed on patients' experiences with preventive interventions and their preferences about receiving these interventions, in order to prevent weight gain and the associated health risks.

No evidence on screening for health issues and excess body weight for those who are obese

Table 2: Summary of recommendations on prevention of weight gain in normal-weight adults and treatment of overweight and obesity in Canada and elsewhere

\begin{tabular}{|c|c|}
\hline Organization & Recommendation \\
\hline \multicolumn{2}{|c|}{ Prevention of weight gain } \\
\hline CTFPHC (2015) & $\begin{array}{l}\text { We recommend that practitioners not offer formal, structured interventions }{ }^{*} \text { aimed at preventing weight } \\
\text { gain in normal-weight adults. }\end{array}$ \\
\hline CTFPHC (2006) $)^{15}$ & $\begin{array}{l}\text { Insufficient evidence to recommend for or against community-wide cardiovascular disease preventive } \\
\text { programs to prevent obesity. Fair evidence to recommend intensive individual and small-group counselling } \\
\text { for a reduced-calorie or low-fat diet to prevent obesity. Fair evidence to recommend an intensive individual } \\
\text { or structured group program of endurance exercise to prevent obesity. Insufficient evidence to recommend a } \\
\text { program of strength-training exercise to prevent obesity. Fair evidence to recommend an intensive individual } \\
\text { or small-group program of a combined low-fat/reduced-calorie diet and endurance exercise intervention to } \\
\text { prevent obesity. Fair evidence to recommend against low-intensity interventions employing telephone or } \\
\text { mail support, or financial incentives to promote a low-fat/reduced-calorie diet and endurance exercise as a } \\
\text { means to prevent obesity. }\end{array}$ \\
\hline USPSTF $(2012)^{46}$ & No prevention recommendation; screen all adults for obesity. \\
\hline NICE $(2010)^{47}$ & $\begin{array}{l}\text { Everyone should aim to maintain or achieve a healthy weight, to improve their health and reduce the risk of } \\
\text { diseases associated with overweight and obesity; people should follow the strategies which may make it } \\
\text { easier to maintain a healthy weight by balancing "calories in" and "calories out." }\end{array}$ \\
\hline SIGN $(2010)^{48}$ & $\begin{array}{l}\text { Individuals consulting about weight management should be advised to reduce intake of energy-dense foods } \\
\text { by selecting foods with low energy density instead, reduce consumption of fast foods and reduce alcohol } \\
\text { intake. Individuals consulting about weight management should be encouraged to be physically active and } \\
\text { reduce sedentary behaviour, including television-watching. }\end{array}$ \\
\hline $\begin{array}{l}\text { Obesity Canada } \\
(2007)^{49}\end{array}$ & $\begin{array}{l}\text { Recommend programs that combine a low-fat or energy-reduced diet and endurance exercise; individual and } \\
\text { small-group counselling for dietary interventions; insufficient evidence for community interventions aimed at } \\
\text { cardiovascular disease risk reduction. }\end{array}$ \\
\hline \multicolumn{2}{|c|}{ Treatment of overweight and obesity } \\
\hline CTFPHC (2015) & $\begin{array}{l}\text { Offer or refer to formal, structured behavioural interventions aimed at decreasing weight in overweight or } \\
\text { obese adults. Do not recommend pharmacologic interventions. }\end{array}$ \\
\hline CTFPHC (1999) $)^{14}$ & $\begin{array}{l}\text { Insufficient evidence to recommend weight-reduction therapy for obese adults without obesity-related } \\
\text { diseases; for obese adults with obesity-related diseases weight reduction is recommended. }\end{array}$ \\
\hline $\begin{array}{l}\text { National Health } \\
\text { and Medical } \\
\text { Research Council } \\
\text { (Australia) }(2013)^{50}\end{array}$ & $\begin{array}{l}\text { For adults who are overweight or obese, strongly recommend lifestyle change }- \text { including reduced-energy } \\
\text { intake, increased physical activity and measures to support behavioural change. } \\
\text { For adults with a BMI } \geq 30 \text {, or adults with a BMI } \geq 27 \text { and comorbidities, orlistat may be considered as an } \\
\text { adjunct to lifestyle interventions, taking into account the individual situation. }\end{array}$ \\
\hline $\begin{array}{l}\text { USPSTF (2012; } \\
2014)^{46,51}\end{array}$ & $\begin{array}{l}\text { Screen all adults for obesity; offer or refer patients with a BMI } \geq 30 \text { to intensive, multicomponent behavioural } \\
\text { interventions. Offer or refer adults who are overweight or obese and have additional cardiovascular disease } \\
\text { risk factors to intensive behavioural counselling interventions to promote a healthful diet and physical } \\
\text { activity for cardiovascular disease prevention. }\end{array}$ \\
\hline $\operatorname{NICE}(2010)^{47}$ & $\begin{array}{l}\text { Multicomponent interventions are the treatment of choice. Weight-management programs should include } \\
\text { behaviour change strategies to increase physical activity or decrease inactivity, improve eating and diet } \\
\text { quality and reduce energy intake. Pharmacologic treatment should be considered only after dietary, exercise } \\
\text { and behavioural approaches have been started and evaluated. }\end{array}$ \\
\hline SIGN $(2010)^{48}$ & $\begin{array}{l}\text { Weight-management programs should include physical activity, dietary change and behavioural components. } \\
\text { Orlistat should be considered as an adjunct to lifestyle interventions in the management of weight loss } \\
\text { following assessment of risk and benefits. }\end{array}$ \\
\hline $\begin{array}{l}\text { Obesity Canada } \\
(2007)^{49}\end{array}$ & $\begin{array}{l}\text { Recommend an energy-reduced diet, regular physical and comprehensive lifestyle intervention. } \\
\text { Pharmacotherapy for adults not attaining or unable to attain weight loss with diet and exercise therapy. }\end{array}$ \\
\hline & $\begin{array}{l}\text { CTFPHC = Canadian Task Force on Preventive Health Care, NICE = National Institute for Health and Clinical Excellence (UK), } \\
\text { Guideline Network, USPSTF = U.S. Preventive Services Task Force. } \\
\text { ins are behavioural modification programs that involve several sessions or interactions that take place over weeks to months. } \\
\text { e behaviourally based prevention interventions focused on diet, exercise, lifestyle changes or any combination of these. }\end{array}$ \\
\hline
\end{tabular}


or overweight was identified. There is insufficient evidence to determine effects on overall health, and patient values and preferences for participation in treatment interventions for overweight and obesity. Implementation studies in primary care are needed to assess short-term comparative effectiveness of the available programs, as well as longer-term studies on health outcomes (well-being, disease incidence and mortality) in overweight/obese adults generally and those at greater health risk, including Aboriginal populations and those living in poverty ${ }^{52}$ Given the limited long-term effectiveness of existing therapies for obesity management, and the evolving field of pharmacotherapy, there is a need for further research on the most effective strategies to address this chronic condition.

\section{Conclusion}

The average weight and BMI of Canadian adults have increased over the last 40 years, with a corresponding increase in the prevalence of obesity. Effective approaches to slow or reverse this increase are unknown. A strong recommendation for height and weight measurement in adults was justified, because errors in estimation have been well documented. We found some evidence of the effectiveness of interventions designed to prevent weight gain and assist in weight maintenance in normal-weight adults. Although these interventions were intensive, they led to a small absolute benefit without evidence of sustained effects. Some individuals with normal weight may still benefit from interventions for weight-gain prevention, such as individuals with metabolic risk factors, high waist circumference, family history of type 2 diabetes or cardiovascular disease, or those who are motivated to avoid weight gain. Research is urgently needed about how best to prevent weight gain in normal-weight adults.

A strong recommendation for weight-loss interventions among people at high risk for diabetes was justified by evidence from several trials of decreased incidence of diabetes. Intensive behavioural interventions lead to small clinical changes in weight in overweight and obese adults, with few to no harms. Pharmacologic plus behavioural interventions also promote weight loss, but can lead to harms. In the judgment of the task force, the differential benefit-toharm ratio associated with these treatments justifies a recommendation in favour of behavioural treatments for modest weight loss, but against routine use of pharmacologic interventions.

Behavioural interventions for overweight and obesity require long-term commitment to change diet and physical activity habits. Values and pref- erences, previous experiences with weight loss, personal motivators and barriers, social support and practical resources will all affect decisions to undertake intervention. Discussion with the patient is needed to determine the best course of action. This guideline has identified what benefits can be expected from weight-loss interventions, based on evidence from clinical trials. Additional work is needed to further develop support mechanisms and counselling tools for primary care practitioners.

\section{References}

1. Obesity in Canada: a joint report from the Public Health Agency of Canada and the Canadian Institute for Health Information. Ottawa: Canadian Institute for Health Information, Public Health Agency of Canada; 2011.

2. Chronic disease indicator framework, Quick Stats 2013 edition. Ottawa: Centre for Chronic Disease Prevention, Public Health Agency of Canada; 2013

3. Canadian health measures survey: cycle 2 data tables, 2009 to 2011. Ottawa: Statistics Canada; 2013. Cat no. 82-626-X.

4. Body composition of Canadian adults, 2009 to 2011. Ottawa: Statistics Canada; 2013. Available: www.statcan.gc.ca/pub/82 -625-x/2012001/article/11708-eng.htm (accessed 2014 Mar. 22).

5. Guh DP, Zhang W, Bansback N, et al. The incidence of co-morbidities related to obesity and overweight: a systematic review and meta-analysis. BMC Public Health 2009;9:88.

6. Ng C, Corey PN, Young TK. Divergent body mass index trajectories between Aboriginal and non-Aboriginal Canadians 1994-2009 - an exploration of age, period, and cohort effects. Am J Hum Biol 2012;24:170-6.

7. Orpana HM, Tremblay MS, Fines P. Trends in weight change among Canadian adults. Health Rep 2007;18:9-16.

8. Tackling obesities: future choices - obesogenic environments evidence review. London (UK): Government Office for Science; 2007. Available: www.bis.gov.uk/assets/foresight/docs /obesity/03.pdf (accessed 2014 Mar. 22).

9. Katzmarzyk PT, Ardern CI. Overweight and obesity mortality trends in Canada, 1985-2000. Can J Public Health 2004;95:16-20.

10. Healthy living. In: Health Canada [website]. Available: www hc-sc.gc.ca/hl-vs/index-eng.php (accessed 2014 Mar. 22).

11. Obesity: preventing and managing the global epidemic. Report of a WHO consultation (WHO Technical Report Series 894). Geneva: World Health Organization; 2000.

12. Pottie K, Jaramillo A, Lewin G, et al. Recommendations on screening for type 2 diabetes in adults. CMAJ 2012;184:1687-96.

13. Stevens J, Truesdale KP, McClain JE, et al. The definition of weight maintenance. Int J Obes (Lond) 2006;30:391-9.

14. Douketis JD, Feightner JW, Attia J, et al. Periodic health examination, 1999 update: 1 . detection, prevention and treatment of obesity. Canadian Task Force on Preventive Health Care. CMAJ 1999;160:513-25.

15. Prevention of weight gain and obesity in adults: a systematic review. Calgary: Canadian Task Force on Preventive Health Care 2006. Available: canadiantaskforce.ca/ctfphc-guidelines/2006weight-gain-and-obesity (accessed 2014 Dec. 11).

16. Peirson L, Fitzpatrick-Lewis D, Ciliska D, et al., editors. Pre vention of overweight/obesity in adult populations: a systematic review with meta-analyses. Ottawa: Canadian Task Force on Preventive Health Care; 2014.

17. LeBlanc E, O'Connor E, Whitlock EP, et al. Screening for and management of obesity and overweight in adults [Internet]. Rockville (MD): Agency for Healthcare Research and Quality (US); 2011. Report no 11-05159-EF-1.

18. Schünemann H, Brozek J, Oxman A, editors. GRADE handbook for grading quality of evidence and strength of recommendation. Version 3.2 [updated March 2009]. The GRADE Working Group; 2009. Available: www.cc-ims.net/gradepro (accessed 2014 Dec. 11).

19. Connor Gorber S, Singh H, Pottie K, et al. Process for guideline development by the reconstituted canadian task force on preventive health care. CMAJ 2012;184:1575-81.

20. Kaczorowski J, Robinson C, Nerenberg K. Development of the CANRISK questionnaire to screen for prediabetes and undiagnosed type 2 diabetes. Can J Diabetes 2009;33:381-5.

21. Lindström J, Tuomilehto J. The diabetes risk score: a practical tool to predict type 2 diabetes risk. Diabetes Care 2003;26:725-31.

22. Peirson L, Douketis J, Ciliska D, et al. Treatment for overweight 
and obesity in adult populations: a systematic review and metaanalysis. CMAJ Open 2014;2:E306-17.

23. Connor Gorber S, Tremblay M, Moher D, et al. A comparison of direct vs. self-report measures for assessing height, weight and body mass index: a systematic review. Obes Rev 2007;8:307-26.

24. Ahern T, Doherty K, Kapeluto D, et al. Body mass index estimation and measurement by healthcare professionals. Open $J$ Prev Med 2012;2:265-71.

25. Keys A, Fidanza F, Karvonen MJ, et al. Indices of relative weight and obesity. Int J Epidemiol 2014;43:655-65.

26. Wells JC. Commentary: The paradox of body mass index in obesity assessment: not a good index of adiposity, but not a bad index of cardio-metabolic risk. Int J Epidemiol 2014;43:672-4.

27. Obesity and overweight. Geneva: World Health Organization; 2011.

28. Plourde G, Prud'homme D. Managing obesity in adults in primary care. CMAJ 2012;184:1039-44.

29. Reis JP, Macera CA, Araneta MR, et al. Comparison of overall obesity and body fat distribution in predicting risk of mortality. Obesity (Silver Spring) 2009;17:1232-9.

30. Douketis JD, Paradis G, Keller H, et al. Canadian guidelines for body weight classification in adults: application in clinical practice to screen for overweight and obesity and to assess disease risk. CMAJ 2005;172:995-8.

31. Després J, Tchernof A. Classification of overweight and obesity in adults. CMAJ 2007;178:21-6.

32. Sharma AM, Kushner RF. A proposed clinical staging system for obesity. Int J Obes (Lond) 2009;33:289-95.

33. Forster JL, Jeffery RW, Schmid TL, et al. Preventing weight gain in adults: a pound of prevention. Health Psychol 1988;7:515-25.

34. Lawton BA, Rose SB, Elley CR, et al. Exercise on prescription for women aged 40-74 recruited through primary care: two year randomised controlled trial. BMJ 2008;337:a2509.

35. Vermunt PW, Milder IE, Wielaard F, et al. A lifestyle intervention to reduce type 2 diabetes risk in Dutch primary care: 2.5-year results of a randomized controlled trial. Diabet Med 2012;29:e223-31.

36. Carty CL, Kooperberg C, Neuhouser ML, et al. Low-fat dietary pattern and change in body-composition traits in the women's health initiative dietary modification trial. Am J Clin Nutr 2011;93:516-24.

37. Knowler WC, Fowler SE, Hamman RF, et al. 10-year follow-up of diabetes incidence and weight loss in the Diabetes Prevention Program Outcomes Study. Lancet 2009;374:1677-86.

38. Barry VW, Baruth M, Beets MW, et al. Fitness vs. fatness on all-cause mortality: a meta-analysis. Prog Cardiovasc Dis 2014; 56:382-90.

39. Blomain ES, Dirhan DA, Valentino MA, et al. Mechanisms of weight regain following weight loss. ISRN Obes 2013; 2013:210524.

40. Brown I, Gould J. Decisions about weight management: a synthesis of qualitative studies of obesity. Clin Obes 2011;1:99-109.

41. Garip G, Yardley L. A synthesis of qualitative research on overweight and obese people's views and experiences of weight management. Clin Obes 2011;1:110-26.

42. Dunkley AJ, Bodicoat DH, Greaves CJ, et al. Diabetes prevention in the real world: effectiveness of pragmatic lifestyle interventions for the prevention of type 2 diabetes and of the impact of adherence to guideline recommendations: a systematic review and meta-analysis. Diabetes Care 2014;37:922-33.

43. Slater J, Green C, Sevenhuysen G, et al. Socio-demographic and geographic analysis of overweight and obesity in canadian adults using the Canadian Community Health Survey (2005). Chronic Dis Can 2009;30:4-15.

44. Dutton DJ, McLaren L. Explained and unexplained regional variation in Canadian obesity prevalence. Obesity (Silver Spring) 2011;19:1460-8

45. The CTFPHC procedure manual. Calgary: Canadian Task Force on Preventive Health Care; 2014.

46. Moyer VA; U.S. Preventive Services Task Force. Screening for and management of obesity in adults: U.S. Preventive Services Task Force recommendation statement. Ann Intern Med 2012; 157:373-8

47. Obesity: the prevention, identification, assessment and management of overweight and obesity in adults and children. NICE clinical guideline 43. London (UK): National Institute for Health and Clinical Excellence; 2006. Available: www.nice.org.uk /CG43 (accessed 2014 Jan. 12).

48. Management of obesity: a national clinical guideline. Edinburgh: Scottish Intercollegiate Guidelines Network; 2010. Available: www.sign.ac.uk/pdf/sign115.pdf (accessed 2014 Jan. 12).

49. Lau DC, Douketis JD, Morrison KM, et al. 2006 Canadian clinical practice guidelines on the management and prevention of obesity in adults and children. CMAJ 2007;176(8 Suppl):Online 1-117.
Available: www.cmaj.ca/content/suppl/2007/09/04/176.8.S1.DC1 /obesity-lau-onlineNEW.pdf (accessed 2014 Jan. 12)

50. Summary guide for the management of overweight and obesity in primary care. Canberra (Australia): Australian Government, National Health and Medical Research Council, Department of Health; 2013.

51. LeFevre ML. Behavioral counseling to promote a healthful diet and physical activity for cardiovascular disease prevention in adults with cardiovascular risk factors: U.S. Preventive Services Task Force recommendation statement. Ann Intern Med 2014;161:587-93.

52. Vallis M, Piccinini-Vallis H, Sharma AM, et al. Clinical review: modified 5 As: minimal intervention for obesity counseling in primary care. Can Fam Physician 2013;59:27-31

Authors: Paula Brauer PhD, Sarah Connor Gorber PhD, Elizabeth Shaw MD, Harminder Singh MD, Neil Bell MD, Amanda R.E. Shane MSc, Alejandra Jaramillo MSc, Marcello Tonelli MD

Competing interests: Paula Brauer is a volunteer member of the Canadian Obesity Network, the Canadian Diabetes Association and Dieticians of Canada. No other competing interests were declared.

Affiliations: Family Relations and Applied Nutrition (Brauer), University of Guelph, Guelph, Ont.; Public Health Agency of Canada (Connor Gorber, Shane, Jaramillo), Ottawa, Ont.; Department of Family Medicine (Shaw), McMaster University, Hamilton, Ont.; Departments of Internal Medicine and Community Health Sciences (Singh), University of Manitoba, Winnipeg, Man.; Department of Family Medicine (Bell) University of Alberta, Edmonton, Alta.; Department of Medicine, University of Calgary (Tonelli), Calgary, Alta.

Contributors: All of the authors contributed substantially to the interpretation of the findings. Paula Brauer, Sarah Connor Gorber and Marcello Tonelli drafted the article with assistance from the rest of the group, and all of the authors gave final approval of the version submitted for publication.

Funding: Funding for the Canadian Task Force on Preventive Health Care is provided by the Public Health Agency of Canada and the Canadian Institutes of Health Research. The views of the funding bodies have not influenced the content of the guideline; competing interests have been recorded and addressed. The views expressed in this article are those of the authors and do not represent those of the Public Health Agency of Canada.

Acknowledgements: The authors acknowledge the authors of the evidence reviews that supported this guideline (Leslea Peirson, Donna Fitzpatrick-Lewis, Mohammed Usman Ali, Donna Ciliska, Rachel Warren, Meghan Kenny, Eva Tsakonas, Maureen Rice, Parminder Raina and James Douketis at McMaster University); Lesley Dunfield at the Prevention Guidelines Division and members of the Health Surveillance and Epidemiology Division at the Public Health Agency of Canada who supported the development of the guideline; and the reviewers whose comments helped to improve this manuscript (Kami Kandola, Council of Chief Medical Officer of Health; Maitreyi Raman, Division of Gastroenterology, Department of Medicine, University of Calgary; Claude Thivierge, Collège des médecins du Québec; Raj Padwal, University of Alberta; Roger Suss, Department of Family Medicine, University of Manitoba; Gilles Plourde, Health Canada; Jessica D. Smith, Harvard School of Public Health; Sharon Kirkpatrick, School of Public Health and Health Systems, University of Waterloo; Joyce Thompson, NHS Tayside, Scotland, UK; David C.W. Lau, Departments of Medicine, Biochemistry and Molecular Biology, Julia McFarlane Diabetes Research Centre and Libin Cardiovascular Institute of Alberta, University of Calgary; Kim D. Raine, Centre for Health Promotion Studies, School of Public Health, University of Alberta; and Rachel Rodin, Public Health Agency of Canada. 